The 1995 Particle Accelerator Conf. \& International Conf. on

High-Energy Accelerators, Dallas, Texas, May 1-5, 1995. $\operatorname{con} f-9505 / 2--122$

\title{
Beam Size Versus Intensity for Resonant Extracted Beam At the Brookhaven AGS*
}

\author{
K.A. Brown and R. Thern, Brookhaven National Laboratory \\ H. Huang, Indiana University
}

\section{Abstract}

Transverse beam sizes were measured in the AGS prior to the initiation of the third order resonance $\left(\nu_{\mathbf{H}}=8 \frac{2}{3}\right.$; driven by sextupoles) used for the slow extraction and again in the external beam lines. The measurements were made using the AGS Ionization Profile Monitor (IPM) and an External Profile Monitor (EPM). Both of these monitors use the ionized residual gas to image the beam on arrays of wires that are aligned parallel to the direction of the beam. The effects of space charge on the IPM are significant at the intensities measured, but the EPM measurements are not affected by space charge. Two measurements are reported. First is the intensity dependence of the resonant beam size, and secondly, an indirect measure of the space charge effect on the IPM. A differential comparison of the two monitors in the vertical plane allows unfolding the effect of the space charge on the measurements. These measurements were made over an intensity range between $15 \times 10^{12}$ and $36 \times 10^{12}$ protons per AGS pulse.

\section{Data Collection}

The AGS IPM is located in the AGS ring and measures beam profiles of the circulating beam. ${ }^{[1]}$ The EPM is located in the transport channel after beam is extracted from the AGS. Both instruments work on the same principle. Residual gas ions are collected on wires which are aligned parallel to the direction of the beam, as the beam passes between two planes of wires at some potential difference. The major difference between the two instuments is that one measures the high current circulating beams while the other measures the low current extracted beam.

The AGS IPM has a dedicated application which interfaces with the AGS controls system to control the device parameters and to collect data. The EPM is only available as an analog signal which can be

*Work performed under the auspices of the U.S. Department of Energy. displayed on an oscilloscope. Using a Lecroy 9404 digital oscilloscope and capturing the data via GPIB into a National Instruments ${ }^{\circledR}$ LabVIEW $^{\$}$ virtual instrument running on a Sun SPARC station IPX, we were able to put together a simple but powerful system which allowed analysis similar to that which is done in the IPM application software. Since we were only interested in getting accurate width and integrated areas of the EPM, we only built the LabVIEW application to do simple least squares analysis of a gaussian distribution on the profiles captured from the Lecroy 9404 . We then captured profiles from the EPM at the same time that we took IPM scans for a series of different intensities.

The beam before it is debunched for extraction has peak currents of as high as $30 \mathrm{~A}$ while after beam is extracted the currents are only a few microampere. The high current bunched beams cause significant space charge effects in the IPM measurement, ${ }^{[2]}$ while the low currents after extraction cause no space charge effects in the EPM measurements. ${ }^{[3]}$

\section{Results}

Figure 1 shows the emittance growth in the vertical plane on the EPM versus intensity. Values shown are of $\epsilon$ (E.G.; area $=0.15 \pi$ ) and are unnormalized. To get normalized emittance multiply by $\beta \gamma=25.7$.

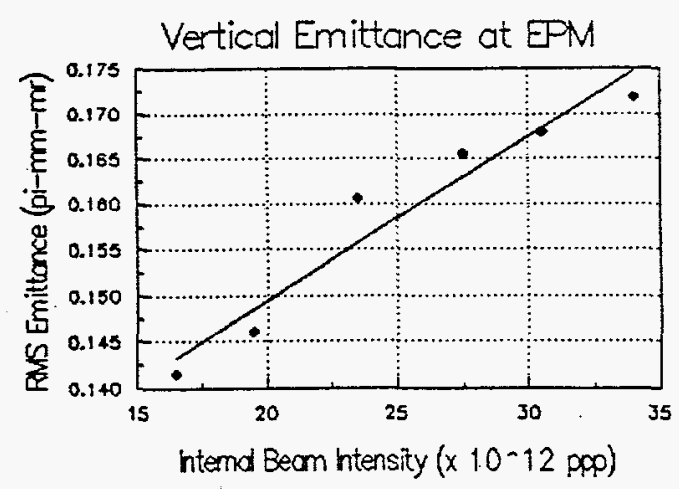

Figure 1: Vertical Emittance vs Intensity 


\section{DISCLAIMER}

This report was prepared as an account of work sponsored by an agency of the United States Government. Neither the United States Government nor any agency thereof, nor any of their employees, makes any warranty, express or implied, or assumes any legal liability or responsibility for the accuracy, completeness, or usefulness of any information, apparatus, product, or process disclosed, or represents that its use would not infringe privately owned rights. Reference herein to any specific commercial product, process, or service by trade name, trademark, manufacturer, or otherwise does not necessarily constitute or imply its endorsement, recommendation, or favoring by the United States Government or any agency thereof. The views and opinions of authors expressed herein do not necessarily state or reflect those of the United States Government or any agency thereof. 


\section{DISCLAIMER}

Portions of this document may be illegible in electronic image products. Images are produced from the best available original document. 
This shows the emittance increasing linearly at a rate of $1.71 \times 10^{-3} \pi \mathrm{mm}-\mathrm{mr} / \mathrm{TP}$ (RMS), or a $20 \%$ increase over a factor of two in intensity.

Figure 2 shows the emittance growth in the horizontal plane at the EPM.

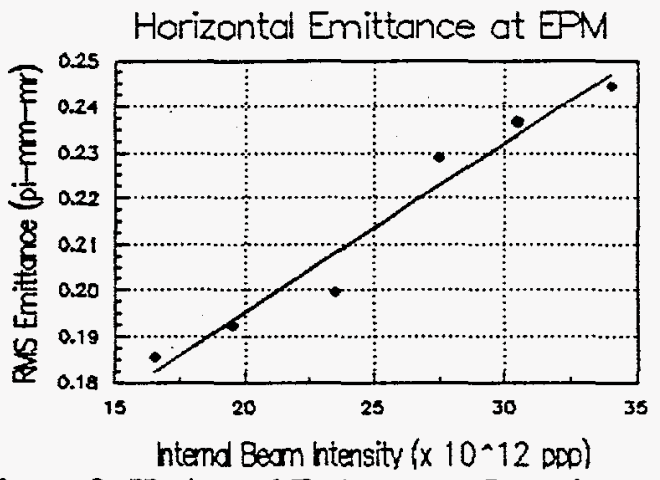

Figure 2: Horizontal Emittance vs Intensity

This shows the emittance increasing linearly at a rate of $3.33 \times 10^{-3} \pi \mathrm{mm}-\mathrm{mr} / \mathrm{TP}$ (RMS), or a $26 \%$ increase over a factor of two in intensity. This measurement represents the growth of the horizontal emittance observed after the extraction process. This growth is roughly twice that seen in the vertical plane, although the percentage increase is about the same.

The uncertainty in the above emittances from the EPM is approximately $\pm 30 \%$. This uncertainty is primarily due to the uncertainty in $\beta$ at the EPM. What is of interest, though, is the relative variations with intensity, which can be measured very well, as seen by the scatter of the points around the straight line in figures 1 and 2.

The IPM shows the vertical beam size changing in the process of debunching the beam for extraction. The IPM measurement suffers substantial distortion from the space charge forces of the beam at these high intensities and small beam sizes. It is believed that the actual vertical size does not change during the debunching process, and thus the size difference is a manifestation of the change in space charge distortion in changing from a bunched beam which is small horizontally to an unbunched beam which is large horizontally (due to the large $\mathrm{dp} / \mathrm{p}$ attained during the debunching).

Figure 3 shows the inferred beam size, where the correction has been made by a Monte-Carlo simulation of the ion collection process in the IPM. At this small beam size and high intensity, the simulation is only feasible for the unbunched beam. For a bunched beams this narrow and intense, the distortion is very sensitive to several parameters that are insufficiently well known to allow a meaningful unfolding of the effect.

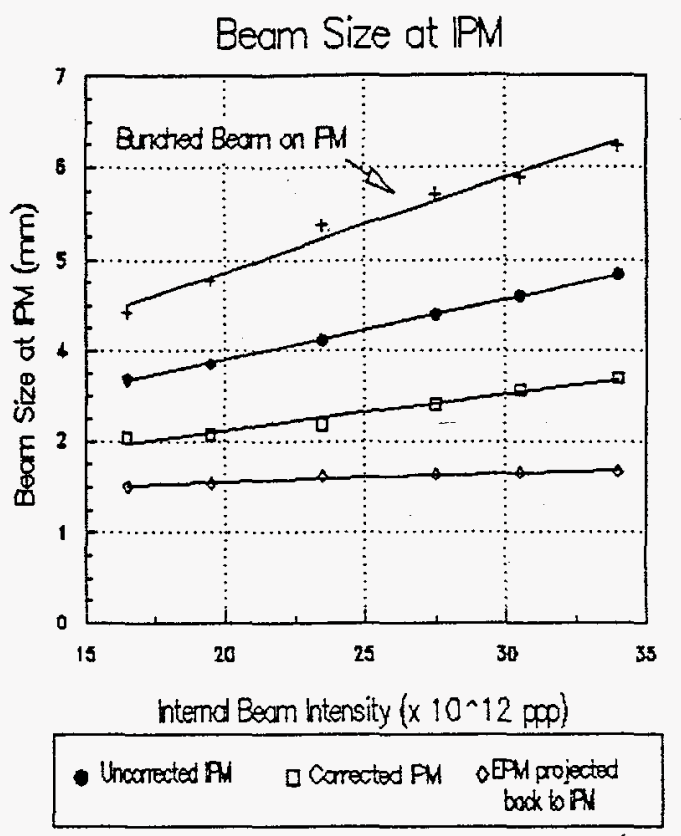

Figure 3: Vertical Beam Size for Debunched Beam versus Intensity.

\section{Conclusions}

The growth in the emittance observed after resonant extraction is approximately 20 to $30 \%$ over a range of intensities of 15 to $35 \times 10^{\wedge} 12$ protons/AGS repitition period, both vertically and horizontally. The AGS IPM also shows a modest growth, but unfolding the actual beam sizes is extremely difficult. In general, though, and given the uncertainties in both instruments, the agreement is very good.

\section{References}

[1] H. Weisberg, E. Gill, P. Ingrassia, and E. Rodger, IEEE Trans. Nucl. Sci., NS-30 (1983) 2179.

[2] R.E. Thern, 1987 Particle Accelerator Conf., Wash., D.C., p. 646.

[3] I.H.Chiang, Private Communication 